Meta

Journal des traducteurs

Translators' Journal

\title{
Retenue, déduction, exemption, exonération
}

\section{Lise Genest}

Volume 27, numéro 2, juin 1982

URI : https://id.erudit.org/iderudit/002876ar

DOI : https://doi.org/10.7202/002876ar

Aller au sommaire du numéro

Éditeur(s)

Les Presses de l'Université de Montréal

ISSN

0026-0452 (imprimé)

1492-1421 (numérique)

Découvrir la revue

Citer cet article

Genest, L. (1982). Retenue, déduction, exemption, exonération. Meta, 27(2),

207-209. https://doi.org/10.7202/002876ar d'utilisation que vous pouvez consulter en ligne.

https://apropos.erudit.org/fr/usagers/politique-dutilisation/ 


\section{ÉTUDES \\ TERMINOLOGIQUES \\ ET LINGUISTIQUES}

\section{RETENUE, DÉDUCTION, EXEMPTION, EXONÉRATION}

Les termes retenue, déduction, exemption et exonération se rapportent au domaine du revenu et de la fiscalité et ils prêtent parfois à confusion en raison du recoupement de leur contenu sémantique. La présente étude vise à leur attribuer une définition qui permette d'établir des distinctions.

Retenue: prélèvement opéré sur le traitement au moment de son versement et effectué en raison de certaines obligations ou en vertu de certaines conventions. Ce retranchement peut s'appliquer au remboursement d'une dette, au paiement d'une pension ou à une cotisation pour sécurité sociale. Le dispositif de retenue à la source constitue également un mode de recouvrement de l'impôt qui permet le prélèvement à la naissance de la créance fiscale. L'amputation est forfaitaire et provisoire dans le domaine de l'imposition.

Déduction : d'une façon générale, la déduction est une simple soustraction ou un retranchement. En fiscalité cependant, il s'agit d'un privilège qui entraîne une réduction effectuée sur l'assiette des impôts ou sur l'impôt lui-même. Les déductions s'appliquent au revenu brut pour en arriver au revenu net. Ainsi, on déduira des revenus bruts les charges qui ont été supportées pour les acquérir (par exemple, les frais professionnels). Les déductions se divisent en deux catégories, soit les charges réelles (pour lesquelles les autorités demandent des justifications) et les charges forfaitaires, qui sont calculées en pourcentage du revenu brut. C'est la législation qui décide d'accorder les déductions; il s'agit d'un moyen d'encourager certaines activités comme l'épargne ou le commerce. Le taux ou le barème d'impôt est appliqué sur l'assiette établie après imputation de toutes les déductions admises.

$\mathrm{Au}$ Québec et au Canada, les déductions qui tiennent compte des charges familiales sont désignées sous le nom d'exemption.

Exemption: privilège qui affranchit d'une charge, d'une obligation. Par exemple, exemption d'impôts, de taxes.

Dans les déclarations d'impôt du Québec et du Canada, on accorde au mot exemption un sens particulier. Dans la catégorie des déductions, l'exemption permet un soulagement fiscal s'appliquant à la charge familiale. À la différence de certaines déductions, les exemptions permises ne concernent pas les frais supportés pour gagner un revenu. Le calcul des exemptions permet le passage du revenu 
net au revenu imposable. Les exemptions sont forfaitaires ou calculées selon certaines variantes.

Exonération: dispense totale ou partielle d'une charge, d'une obligation financière. Tout comme la déduction et l'exemption, l'exonération a le sens de "réduction d'impôt consentie par la loi». Toutefois, l'usage en fiscalité canadienne et québécoise lui confère un contenu sémantique plus restreint. Ainsi, dans la Loi de l'impôt sur le revenu, son utilisation semble se limiter aux non-résidents qui sont exonérés en totalité. Par conséquent, les termes déduction et exemption devraient s'appliquer aux cas concrets où des retranchements sont effectués. Par ailleurs, on réservera le terme exonération à un usage plus général et non technique lorsqu'il n'est pas question des non-résidents. On parlera par exemple d'exonération d'impôt ou on dira d'un contribuable qu'il est exonéré.

Synonymes d'exonération: abattement, décharge, dégrèvement, diminution, dispense, exemption, remise.

Exemples de déductions et d'exemptions possibles

\section{DÉDUCTIONS}

\section{EXEMPTIONS}

- contributions à un régime enregistré d'épargne-logement (R.E.E.L.)

- cotisations à un régime enregistré de pensions

- cotisations au Régime de rentes du Québec (R.R.Q.)

- cotisations syndicales, professionnelles ou semblables

- déduction forfaitaire de $100 \$$

- exemptions

- frais de déménagement

— frais de garde d'enfants

- frais de scolarité

- frais médicaux

- primes d'un régime enregistré d'épargne-retraite (R.E.E.R.)

- exemption de personne mariée

- exemption pour enfants et autres personnes à charge

- exemption personnelle de base

(Tiré du Guide général d'impôt 1980 - résidents du Québec, Revenu Canada Impôt.)

Aide-mémoire

Retenue : retranchement opéré sur le salaire à son versement.

Déduction: imputation de certains frais ou de dépenses, appliquée au revenu brut.

Exemption: déduction qui concerne les charges familiales.

Exonération: décharge d'une obligation financière en partie ou en totalité. En fiscalité québécoise et canadienne, privilège accordé aux non-résidents, qui les exempte de l'imposition. 


\section{BIBLIOGRAPHIE}

Académie des sciences commerciales (1979) : Dictionnaire commercial, Paris, Hachette.

AZEMA, J. et al. (1974) : Lexique de termes juridiques, Paris, Dalloz, $376 \mathrm{p}$

BAUDHUIN, F., (1968): Dictionnaire de l'économie contemporaine, Verviers, Editions Gérard \& $\mathrm{C}^{\circ}, 301 \mathrm{p}$.

BERNARD, Y. et J.-C. COLLI (1975) : Dictionnaire économique et financier, Paris, Éditions du Seuil, 1168 p.

Centre international du droit des affaires CIDA (1973): Lexique commercial, Paris, Éditions REGIF, $451 \mathrm{p}$.

Conseil international de la langue française (1972): Vocabulaire de l'administration, Paris, Hachette, $187 \mathrm{p}$.

MAGNET, J. (1980) : Lexique - droit budgétaire et comptabilité publique, Paris, P.U.F.

ROYER, P. et J. DREW (1979): Impôt et planification, Montréal, Editions sciences et culture inc..

Dictionnaire de la comptabilité (1980), Paris, Dictionnaires fiduciaires.

Ouvrages gouvernementaux

Loi de l'impôt sur le revenu du Canada.

Revenu Canada Impôt, Guide général d'impôt 1980 - résidents du Québec, 52 p. 